\title{
Distributed Sensor Networks for Health Monitoring of Civil Infrastructures
}

\author{
Ting-Hua Yi, ${ }^{1}$ Gangbing Song, ${ }^{2}$ Stathis C. Stiros, ${ }^{3}$ and Bo Chen ${ }^{4}$ \\ ${ }^{1}$ School of Civil Engineering, Dalian University of Technology, Dalian 116023, China \\ ${ }^{2}$ Department of Mechanical Engineering, University of Houston, Houston, TX 77204-4006, USA \\ ${ }^{3}$ Department of Civil Engineering, Patras University, 26500 Patras, Greece \\ ${ }^{4}$ School of Civil Engineering and Architecture, Wuhan University of Technology, Wuhan 430070, China \\ Correspondence should be addressed to Ting-Hua Yi; yth@dlut.edu.cn
}

Received 4 March 2015; Accepted 4 March 2015

Copyright ( $) 2015$ Ting-Hua Yi et al. This is an open access article distributed under the Creative Commons Attribution License, which permits unrestricted use, distribution, and reproduction in any medium, provided the original work is properly cited.

Structural Health Monitoring (SHM) implies the integration of nondestructive evaluation methods within a system to enable autonomous state awareness for structural integrity. SHM systems have been optimized to cover wide areas as well as focus on specific "hot-spots" for both metallic and composite structures. They can be configured to monitor adverse "changes" such as fatigue cracks, corrosion, delamination, loose bolts, or impact damage either in real-time or on demand. Immediate benefits of SHM include drastically reducing inspection costs, minimizing preventative maintenance, increasing asset availability, and extending remaining useful life of structures. Future benefits could include using SHM data to improve design-margin efficiency for lighterweight structures, facilitating structural certification and/or quality assurance, and dynamically controlling operating envelopes. Over the past few years, there has been rapid development of SHM technique; many smart sensors and intelligent algorithms have been developed. Advances in sensing technologies have also enabled the use of large numbers of sensors for health monitoring of civil infrastructures. The sensors utilized in a SHM system are required to monitor not only the structural status including stress, displacement, and acceleration, but also those influential environmental parameters, such as wind speed, temperature, and the quality of its foundation. Since a large-scale sensor network needs to be involved in a SHM system, how to deploy it in a complicated, spatially extended structure and the resulting best identification of structural characteristics by the data acquired from those locations will be challenging tasks.

Therefore, in the light of the above considerations, this special issue was launched. Numerous investigators worldwide were invited to contribute their original papers and review articles on the theme of this special issue. These papers present the most recent advances, progress, and ideas in the field of distributed sensor networks for SHM and its application includes smart, bioinspired, nanometer, wireless, and remote sensing technology; intelligent algorithms and evaluation criteria in design of sensor networks; uncertainty, sensitivity, reliability, and redundancy in vibration monitoring; vibration data cleaning, compression, mining, and fusing technology; application of large-scale sensor networks for civil infrastructures. In the paper "Fatigue Performance Assessment of Composite Arch Bridge Suspenders Based on Actual Vehicle Loads" by B. Chen et al., the fatigue conditions of bridge suspenders are analyzed according to the Palmgren-Miner linear fatigue cumulative damage criterion. In the paper "Sensor Placement Optimization of Vibration Test on Medium-Speed Mill" by L. Zhu et al., the optimal sensor placement of medium-speed mill is conducted using the effective independence method and QR decomposition algorithm. The results showed that the order of modal shapes had an important influence on the optimization results. The paper "Feasibility Verification of Mountable PZT-Interface for Impedance Monitoring in Tendon-Anchorage" by T.C. Huynh et al. numerically evaluated the performance 
of the mountable PZT-interface for impedance monitoring in tendon-anchorage. In the paper "Effect of Temperature Variation on Vibration Monitoring of Prestressed Concrete Girders" by T.-C. Huynh et al., the effect of temperature variation on vibration monitoring of prestressed concrete girders is experimentally analyzed. The paper "Evaluation on Impact Interaction between Abutment and Steel Girder Subjected to Nonuniform Seismic Excitation" by Y. Zheng et al. investigates the pounding interaction between the abutment and the steel girder underground motions for structural performance assessment. In the paper "The LongTerm Settlement Deformation Automatic Monitoring System for the Chinese High-Speed Railway" by X. Wang et al., using the newly built Shijiazhuang-Jinan passenger railway as an example, the authors developed a set of online monitoring systems, which were automatic, real-time, remote, and visual, and they were suited to the standards of the Beijing-Shanghai high-speed railway. The paper "Impact Coefficient Analysis of Long-Span Railway Cable-Stayed Bridge Based on Coupled Vehicle-Bridge Vibration" by Y. Li et al. discusses the impact effects on main girder, stayed cable, bearings, and bridge tower at various vehicle speeds. The paper "Running Safety of Trains under Vessel-Bridge Collision" investigates the characteristics of a train-bridge system under vesselbridge collision. The paper "Health Status Monitoring of Cuplock Scaffold Joint Connection Based on Wavelet Packet Analysis" by L. Zhang et al. describes how to use the piezoelectric transducers to monitor cuplock connection for cuplock scaffold based on the wavelet packet analysis. The paper "Study Based on Bridge Health Monitoring System on Multihazard Load Combinations of Earthquake and Truck Loads for Bridge Design in the Southeast Coastal Areas of China" by D. Sun et al. presents a method to combine earthquake load and truck load in the service life of bridges. The paper "Embedded Decentralized Modal Identification of Four-Story Benchmark Structure Using Smart Sensor Network" by A. Y. Fallah et al. proposes a two-step algorithm for system identification of structures using wireless sensing network with decentralized data acquisition strategy. In the paper "Acoustic Emission Signal Recognition of Different Rocks Using Wavelet Transform and Artificial Neural Network" by X. Liu et al., the wavelet transform and artificial neural network is applied to determine rock types from their AE characteristic parameters. The paper "Probability Model of Hangzhou Bay Bridge Vehicle Loads Using Weighin-Motion Data" by D. Sun et al. performs a statistical analysis of the vehicle loads on Hangzhou Bay Bridge using more than 3 months of weigh-in-motion data from the site. The paper "Anchor Dragging Analysis of Rock-Berm Using Smoothed Particle Hydrodynamics Method" by J. Woo et al. presents dynamic responses of rock-berm structural system under anchor dragging and accordingly provides the characteristics of the stresses and displacements obtained. The paper "A Wireless Strain Sensor Network for Structural Health Monitoring" by C. Liu et al. presents the design and characterization of a new wireless strain sensor board. The results of the characterization of the fabricated device in combination with IRIS mote show very good performance with respect to the devices' static, sensitivity, and dynamical characteristics. The paper "Research on Impact Process of Lander Footpad against Simulant Lunar Soils" by B. Huang et al. investigates the interaction between lunar soil simulants and the lander footpad during the impact process using subscale model tests based on prototype vertical impact test apparatus. The paper "Life-Cycle Monitoring of LongSpan PSC Box Girder Bridges through Distributed Sensor Network: Strategies, Methods, and Applications" by Z. Chen et al. proposes a life-cycle monitoring strategy to well capture the behavior of the bridge during its whole life and to maximize the use of sensors. In the paper "Detection on Structural Sudden Damage Using Continuous Wavelet Transform and Lipschitz Exponent" by B. Chen et al., a detection approach based on the CWT is proposed in terms of the decomposed detailed coefficients of continuous wavelet transform to detect the damage time instant and location. The Lipschitz exponent is mathematically used to estimate the local properties of certain function and is applied to reflect the damage severity. The paper "Estimation of Evolutionary Spectra of Monitored Seismic Ground Motions by Transformation of Correlation Functions" by Z. Xu et al. proposes a method for the estimation of evolutionary power spectral density of a nonstationary process by transforming the correlation functions of its sample time histories. In the paper "Seismic Fortification Analysis of the Guoduo Gravity Dam in Tibet, China" by P. Lin et al., a dam seismic failure risk control method is proposed based on the cracking mechanism induced by the dynamic load to ensure dam safety and stability. The paper "Effects of Concrete on Propagation Characteristics of Guided Wave in Steel Bar Embedded in Concrete" by Z. Zheng and Y. Lei investigates dispersion equations of longitudinal wave propagation in reinforced concrete member for the purpose of monitoring steels embedded in concrete. In the paper "Establishment and Application of the Wind and Structural Health Monitoring System for the Runyang Yangtze River Bridge" by H. Wang et al., the establishment of the Wind and Structural Health Monitoring System (WSHMS) implemented on the Runyang Yangtze River Bridge (RYRB) in China is introduced. The paper "Free-Interface Modal Synthesis Based Substructural Damage Detection Method" by S. Chen et al. proposes a free-interface modal synthesis based substructure sensitivity method for damage detection. The paper "Early-Warning Method of Train Running Safety of a High-Speed Railway Bridge Based on Transverse Vibration Monitoring" by Y.-L. Ding et al. presents an early-warning method of train running safety of the high-speed railway bridge by adopting principal component analysis (PCA) method.

Without a doubt, the papers reflect the state-of-the-art researches and developments of this subject.

\section{Acknowledgments}

As the Guest Editors of the special issue, we would like to express our sincere appreciation to all the authors who contributed their work to this exciting special issue. The Guest Editors also thank the reviewers for their valuable and insightful comments that greatly benefited the improvement 
of paper quality. This meaningful work was partially supported by the 973 Program (Grant no. 2015CB060000), the National Natural Science Foundation of China (Grant nos. 51421064, 51478081, 51222806, and 51178083), and the Science Fund for Distinguished Young Scholars of Dalian (Grant no. 2014J11JH125).

Ting-Hua Yi Gangbing Song Stathis C. Stiros Bo Chen 

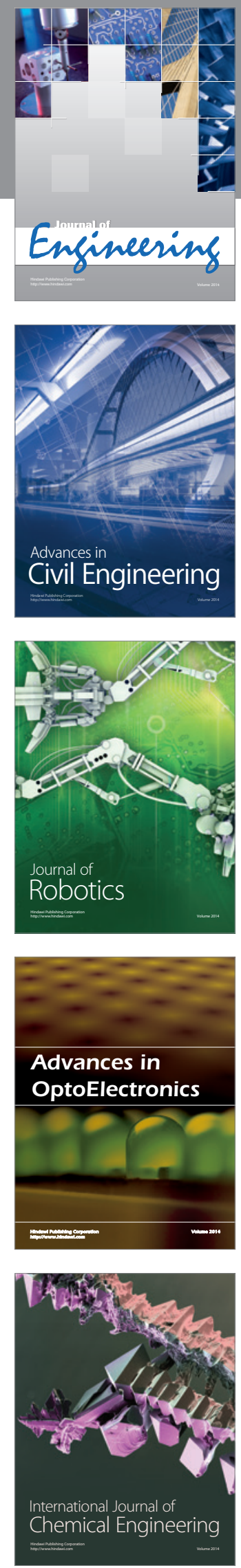

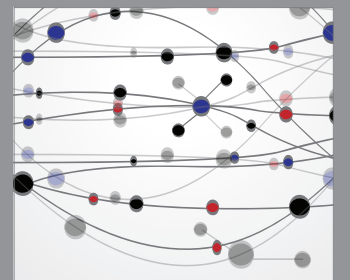

The Scientific World Journal
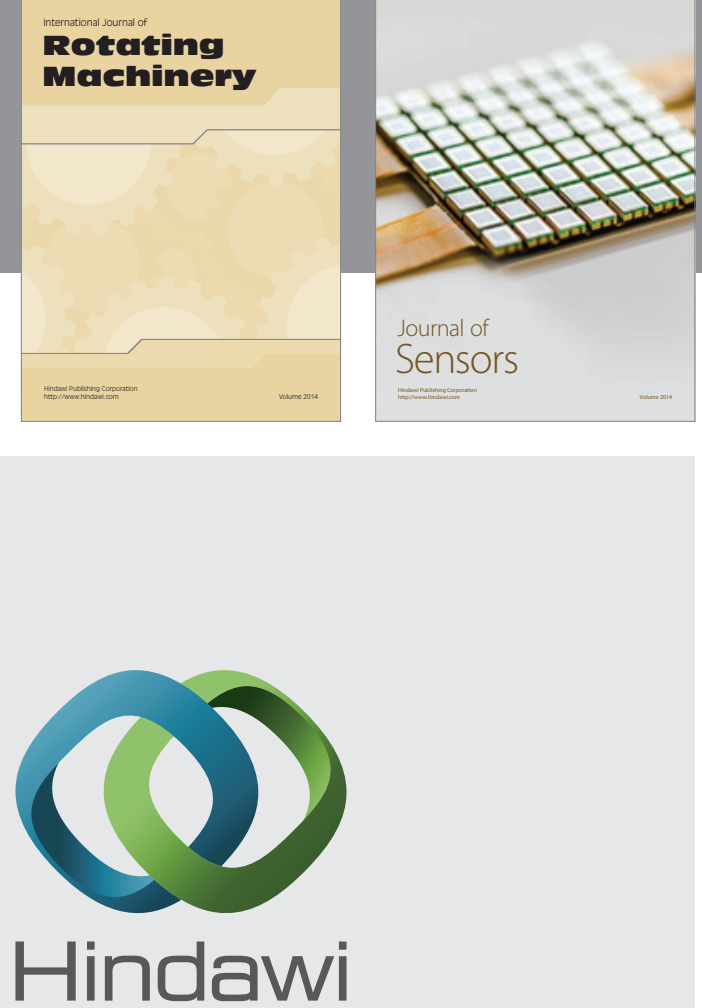

Submit your manuscripts at http://www.hindawi.com
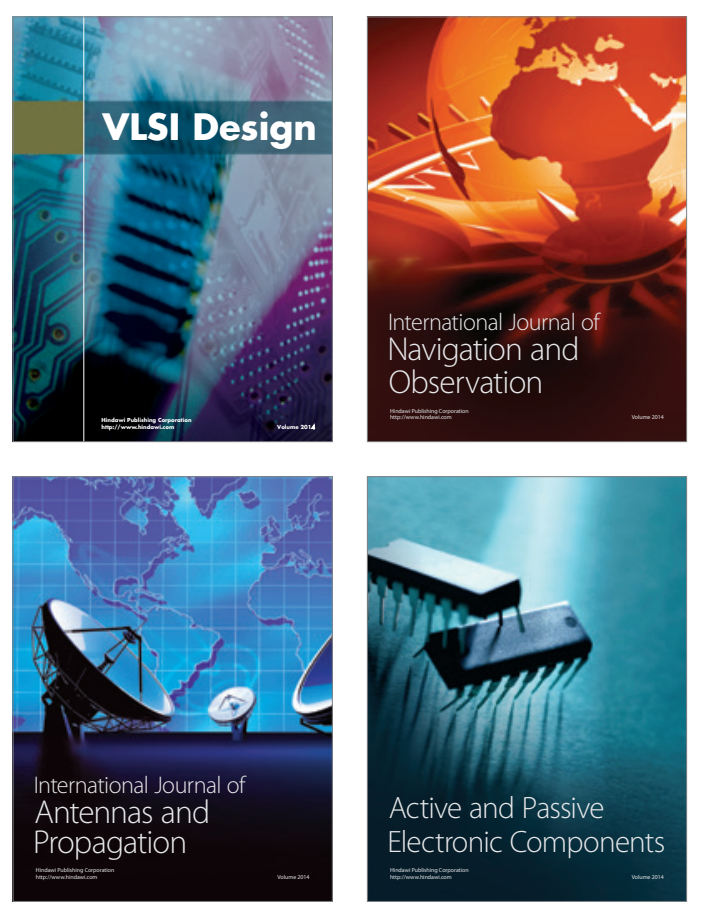
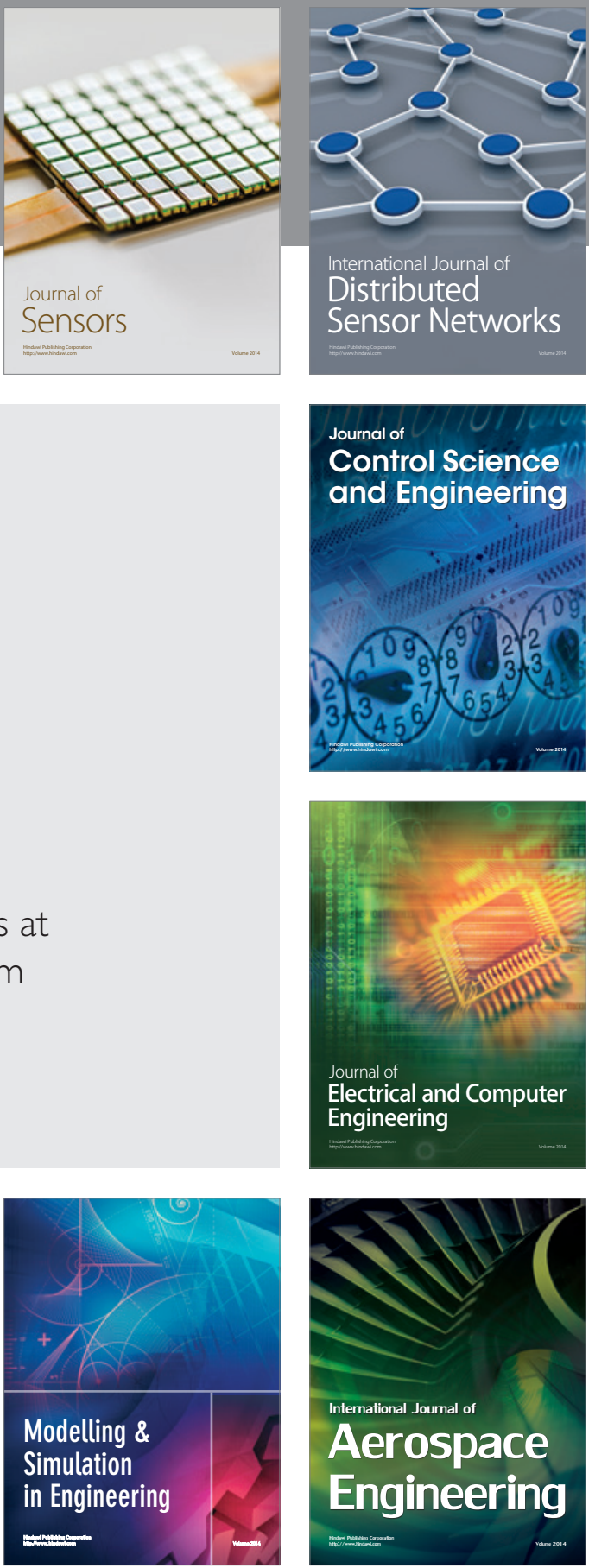

Journal of

Control Science

and Engineering
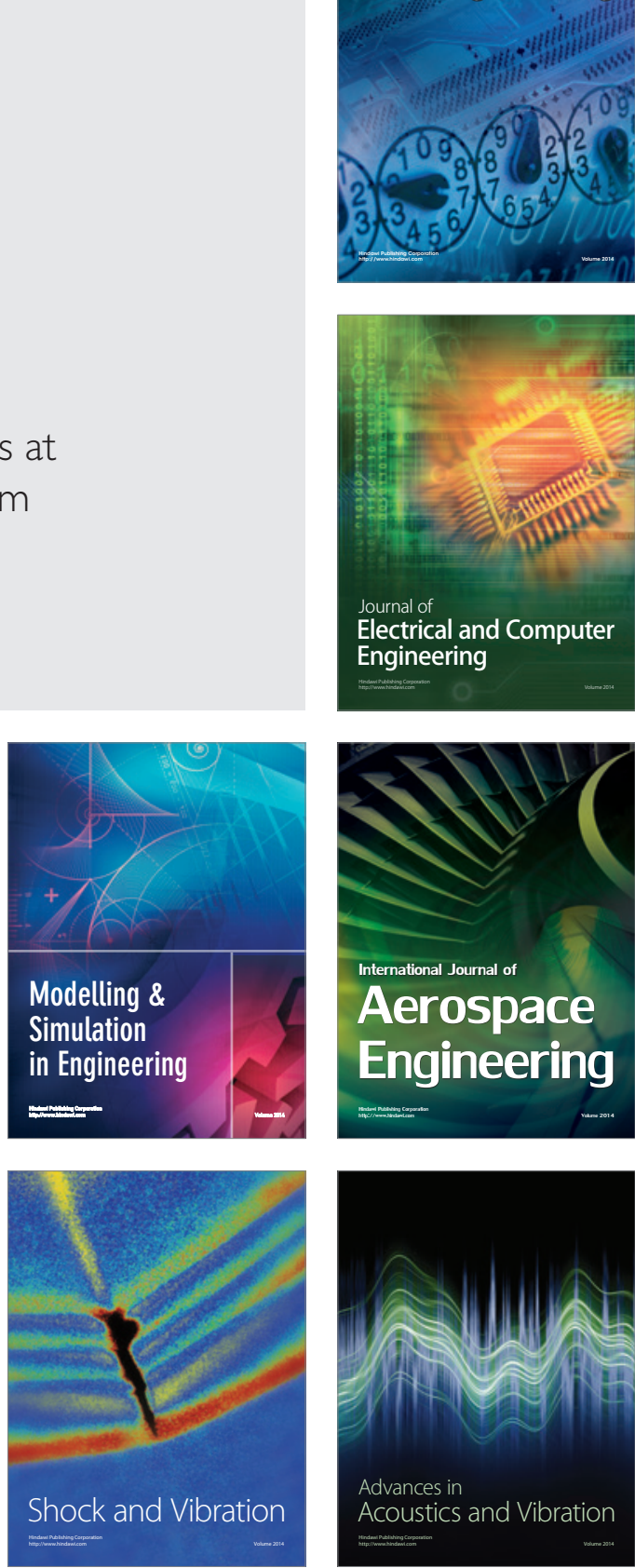